\title{
Mining Cross-Frequency Coupling Microstates (CFC $\mu$ states) from EEG recordings during resting state and mental arithmetic tasks
}

\author{
Stavros I. Dimitriadis*, Yu Sun, Member, IEEE, Nitish Thakor, Fellow, IEEE and Anastasios Bezerianos, Senior Member, IEEE
}

\begin{abstract}
The analysis of functional brain connectivity has been supported by various techniques analyzing the spatiotemporal interactions between distinct brain areas. A large part of brain connectivity studies have analyzed synchronization between dynamic oscillations of identical frequency or between different frequencies. It has been hypothesized that crossfrequency coupling (CFC) between different frequency bands is the carrier mechanism for the coordination of global and local neural processes and hence supports the distributed information processing in the brain. This is the first time that CFC patterns were analyzed via the notion of microstates ( $\mu$ states).

Numerous studies has shown that even at rest human brain activity shows coherent temporal variability and a high degree of complexity. In the present study, we attempt to study the dynamic evolution of CFC at resting-state and while the subjects performing a mental task. For that reason, we introduce here the concept of CFC microstates (CFC $\mu$ states) as emerged short-lived patterns of $\mathrm{CFC}$ resulting from the dynamic variations of coordinated brain activity. Modifying a well-established framework for mining brain dynamics, we show that a small sized repertoire of $\mathrm{CFC} \mu$ states can be derived so as to encapsulate connectivity variations and further provide novel insights into network's functional re-organization.

We analyzed dynamic CFC (dCFC) at resting-state and during a comparison task by adopting a phase-amplitude coupling (PAC) estimator for [ $\delta$ phase- $\gamma$-amplitude] coupling at every sensor. By analyzing the transition dynamics among CFC $\mu$ states, in both tasks, we provided a clear evidence about intrinsic networks that may play a crucial role in information integration.
\end{abstract}

Keyword: cross frequency coupling, resting state, delta, gamma, integration, microstates, default mode network,hierarchical organization

\section{INTRODUCTION}

Many studies have explored changes of brain activity at resting-state in both amplitude an frequency content [1,2]. Neural activity can be recorded non-invasively with electroengephalography (EEG) and magnetoencephalography (MEG) with a high temporal resolution. Even at resting state human brain shows temporal synchronization activity and a high degree of complexity [1,2]. Connectivity at resting state and during various cognitive tasks has been the core of many studies by adopting different methodological approaches [38].

A large body of studies attempted to characterize communication between distinct brain regions within the same frequency component $[4,5,7,8]$. However, there is clear

S. I. Dimitriadis is with Institute of Psychological Medicine and Clinical Neurosciences, Cardiff University School of Medicine and the Cardiff University Brain Research Imaging Center (CUBRIC), School of Psychology, Cardiff University, Cardiff, United Kingdom; AIIA Laboratory, Department of Informatics, and the NeuroInformatics Group, Aristotle University of Thessaloniki, Greece (email: DimitriadisS@cardiff.ac.uk / stidimitriadis@gmail.com ).

Yu Sun (email:kissalladin@gmail.com ),N.Thakor (email: sinapsedirector@gmail.com ) and A. Bezerianos (email: tassos.bezerianos@nus.edu.sg ) are with SINAPSE, National University of Singapore, 28 Medical Drive, 117456, Singapore. evidence that cross-frequency coupling (CFC) is the mechanism that integrates the information between distributed brain networks $[9,10]$.

The microstates literature focused on prototyping (summarizing) spatiotemporal dynamics based on amplitude with a broadband frequency and its relation with cognitive processes [11]. Recently, functional connectomics has becoming a very active research topic and the study of involved dynamics is gaining increasing popularity [7,8,1214]. Lately, an efficient method for handling a huge volume of functional connectivity graphs (FCGs) was introduced [7]. Here, we attempt to detect task differences in terms of CFC through prototyping of CFC emerged patterns .

Our principal goal was to analyze dynamic CFC (dCFC) patterns at resting-state and during a mental task. We estimated dCFC interactions, at every recording site, between $\delta$ phase and $\gamma$ amplitude at resting state and during arithmetic comparison. A phase-amplitude estimator was employed for that purpose [15]. By adopting a recently introduced methodology [7], we summarized the observed CFC variations in each task separately by means of prototypical CFC patterns called hereafter CFC microstates (CFC $\mu$ states).

\section{MATERIALS \& METHODS}

\section{A. Subjects}

In the present study we analyzed recordings from 16 right- handed young volunteers ( 9 males and 7 females, aged:21-26 years, with a mean age of 21.5 (S.D.=1.5) years), who were recruited from the National University of Singapore. All of the participants signed an informed consent form after the procedures had been explained to them. All subjects had normal or corrected-to-normal vision and reported no history of verbal or non-verbal learning disability. The Institutional Review Board of the National University of Singapore approved the procedures [16,17].

\section{B. Experimental Protocol}

The details of the experiment have been described previously $[16,17]$. The task consisted of mentally summing two numbers presented on the computer screen and comparing the result to a proposed answer, also presented on screen. There were 5 possible cognitive workload levels (CWLs). At level 1 problems consisted of summing two one-digit numbers (e.g.,3+8), and each subsequent level included an extra digit so that at level 5 the problems consisted of summing two three-digit numbers (e.g., 471+265). The experiment was segmented in blocks of one minute, with a 30 -second rest period between each block. In the present study, we focused on first restingstate period of 5 mins before the $1^{\text {st }}$ level math task and the $5^{\text {th }} \mathrm{CWL}$ (see Figure 1). 


\begin{tabular}{|c|c|c|c|c|}
\hline Math Task & \multicolumn{2}{|c|}{$\begin{array}{c}\text { Relaxation } \\
\text { (5-min) }\end{array}$} & Math Task & $\begin{array}{c}\text { Relaxation } \\
\text { (5-min) }\end{array}$ \\
\hline Block1 & \multicolumn{3}{|c|}{ Block2 } & \\
\hline $\begin{array}{l}\text { Level } 2 \\
\text { (1-min) }\end{array}$ & $\begin{array}{c}\text { Rest } \\
\text { (30-sec) }\end{array}$ & $\begin{array}{l}\text { Level } 4 \\
\text { (1-min) }\end{array}$ & \begin{tabular}{|c|}
$\begin{array}{c}\text { Rest } \\
\text { (30-sec) }\end{array}$ \\
\end{tabular} & $-\overline{(18 \text { blocks })}$ \\
\hline $26+8$ & pace bar & 33 & $\underset{33<34}{\stackrel{L}{L}}$ & $4+4$ \\
\hline
\end{tabular}

Figure 1. Sequence of events in the mental arithmetic task. Blocks of stimulus (arithmetic level) were presented in a random order

\section{EEG Recordings}

EEG data were recorded from 64-channel active-electrodes, sampled at $256 \mathrm{~Hz}$ with an ActiveTwo Biosemi system (Biosemi Inc., Amsterdam, Netherlands), and referenced using the average reference technique. Bipolar electrooculogram (EOG) was recorded from the outer canthi of both eyes (HEOG) and above and below the left eye (VEOG). Artefacts due to eye blinks or muscle activities during the recordings were removed offline via an independent component analysis (ICA) approach. After concatenating the trials, we used EEGLAB [18] to zero the components that were associated with artefactual activity from eyes, muscle, and cardiac interference. The estimated mixing matrix was used to reconstruct the multichannel signal from the rest independent components

\section{TIME VARYING PAC PATTERNS}

\section{A. Estimating Phase-Amplitude (PAC)Interactions}

Phase-amplitude coupling was estimated within each EEGsensor separately. In order to compute PAC between the phase of a lower frequency $\left(\varphi_{\mathrm{LF}}\right)$ and the amplitude of a higher frequency $\left(\mathrm{A}_{\mathrm{HF}}\right)$, we first extracted the $\mathrm{A}_{\mathrm{HF}}$ and filtered this amplitude time series at the same frequency as LF, giving us the LF modulation in HF amplitude. We then extracted the phase of both the LF signal $\left(\varphi_{\mathrm{LF}}\right)$ and the LF from the amplitude of HF ( $\left.\varphi_{\mathrm{HF}, \mathrm{LF}}\right)$ and computed the phase-locking between these two signals as shown in eq.1 [15,19].

$$
P A C=\frac{1}{N} \sum_{n=1}^{N} \exp \left(i\left(\phi_{L F}(n)-\phi_{H F, L F}(n)\right)\right)
$$

where $\varphi_{\mathrm{LF}}$ denotes the phase of the LF and $\varphi_{\mathrm{HF}, \mathrm{LF}}$ the phase of the LF-filtered from HF amplitude. This phase-locking represents the comodulation of HF amplitude by the phase of LF.

\section{B. Time-Varying CFC (TVCFC)}

By adopting a dynamic approach, a temporal-window of frequency-dependent width was adopted following a sliding mode. With a time step of five samples, the center of the window was moved forward, and the sensor PAC was recomputed based on the new signal segments leading to TVCFC estimates (time-varying cross-frequency coupling). We selected the width of window to be equal to 2 cycles of the $\delta$ activity.

\section{Surrogate Control Analysis of PAC}

To access the significant PAC, we adopted a statistical surrogate analysis which can be performed for each PAC value to infer whether the observed value differs from by an observation by chance. To detect significant PAC interactions, we followed a well-known technique described in [20]. The null hypothesis $\mathrm{H}_{0}$ of the test is that the original PAC value differed significantly from the distribution of surrogates PAC, $\mathrm{PAC}_{\mathrm{s}}$.

For each signal segment and sensor, we form a distribution of 1000 PACs that were generated with the following steps:

step_i: Generate a surrogate time-series $\phi_{L F}^{S}(t)$ by cutting the phase trial of the $\operatorname{LF} \varphi_{\mathrm{LF}}(\mathrm{t})$ in a random selected time window and then exchange the two generated segments. With this approach, we preserve the non-stationarity compared to shuffling the time series.

step_ii: Estimate the surrogate PAC, PAC

We can then determine an one-sided $p$-value for each PAC value that corresponds to the likelihood that the actual observed value. This can be done by directly estimating the proportion of "surrogated" PACs that are higher than the observed PAC [20].

\section{Mining $\mu$ states $^{C F C}$ from TVCFC patterns}

By collecting the quasi-instantaneous PAC measurements, from all the $\mathrm{N}_{\text {sensors }}=64$ sensors, in time-indexed multidimensional patterns of the form $\mathrm{P}(\mathrm{t})=\left[\mathrm{PAC}_{1}(\mathrm{t}), \ldots\right.$ , $\left.\mathrm{PAC}_{64}(\mathrm{t})\right]$, we attempted to characterize the dynamic behavior of CFC over the whole cortex. Our approach involved an unsupervided learning strategy for detecting consistent PAC patterns (PAC $\mu$ states) and a discretization step for assigning the dominant PAC $\mu$ state to every latency. The algorithmic steps were adopted from a recently published paper and are described briefly below $[7,21]$.

In order to summarize the PAC variations, in a group-level analysis, we collected the $\mathrm{P}(\mathrm{t})$ patterns from all the subjects and created a data matrix $\mathbf{P}$ with dimensions equal to [ $\mathrm{N}_{\text {sensors }} \mathbf{X}$ $\left.\mathrm{N}_{\text {Total }}\right]$ where $\mathrm{N}_{\text {sensors }}$ denotes the number of sensors and $\mathrm{N}_{\text {Total }}$ $=\left(\mathrm{N}_{\text {segments }} \mathrm{x} \mathrm{N}_{\text {Subjects }}\right)$.Then, we adopted a vector-quantization step based on Neural Gas algorithm [22], and derived a small number $\mathrm{k}<<\mathrm{N}_{\text {Total }}$ of $64 \mathrm{D}$ prototypes $\left\{\Pi_{\mathrm{j}}\right\}_{\mathrm{j}=1: \mathrm{k}}$. Finally, we assigned each pattern $\mathrm{P}(\mathrm{t})$ to the nearest one among the set of prototypes.

The quality of the adopted encoding scheme was estimated by the normalized distortion error which was defined by the following equation:

$n_{\text {Distortion }}=\frac{\sum_{i=1}^{N_{\text {Towl }}}\left\|\mathrm{P}(\mathrm{t})-\mathrm{P}_{\text {rec }}(t)\right\|^{2}}{\sum_{i=1}^{N_{\text {Tout }} \|}\|\mathrm{P}(\mathrm{t})-\bar{P}\|^{2}}, \quad \bar{P}=\frac{1}{N_{\text {Total }}} \sum_{i=1}^{N_{\text {Tout }}} \mathrm{P}(\mathrm{t})$

where matrix Prec contains the approximated CFC related timeseries produced by replacing the original CFC patterns with the nearest prototypes (CFC $\mu$ states). The smaller the $\mathrm{n}_{\text {Distortion, }}$ the better the encoding. In the present study, we selected the number of PAC $\mu$ states $\mathrm{k}$, as the smallest size of codebook that resulted to $\mathrm{n}_{\text {distortion }}$ below $4 \%$.

\section{RESULTS}

We studied CFC at resting-state and in comparison task based on $\delta$-phase and $\gamma$ amplitude. $\delta$ activity was defined at $(1-4$ $\mathrm{Hz}$ ). In order to assess phase relationship between $\delta$ - phase 
and $\gamma$ amplitude, we bandpass-filtered the EEG data within $(30-45 \mathrm{~Hz})$.

\section{A. states $^{C F C}$ at Resting-State and during an arithmetic task}

We data mined prototypical CFC patterns independently for each recording condition. We then studied the time course of quantified their occupancy and the preferable transition between them. Figure 2 exemplains these data-analytic steps using CFC $^{\text {PAC }}$ patterns associated with brain activity, at resting-state.

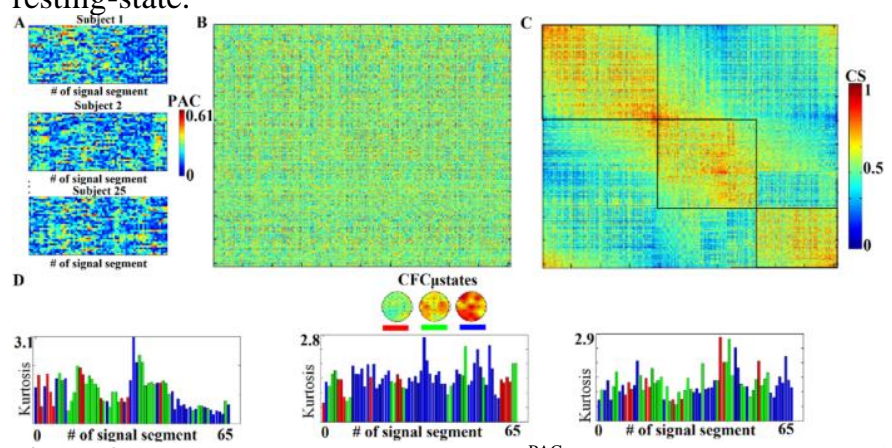

Figure 2. Encoding the time-indexed $\mathrm{CFC}^{\mathrm{PAC}}$ patterns as a sequence of $\mu$ states $^{\mathrm{CFC}}$ : an example using $\mathrm{PAC}^{\delta-\gamma}$ estimates during the resting-state a)Vectorial Time Series of $\mathrm{PAC}^{\delta-\gamma}$ for three subjects. b) The rectangular matrix (RM) containS all the pairwise comparisons (via cosine similarity) among the $\mathrm{N}_{\text {Total }}$ vectors $\mathrm{P}(\mathrm{t})$ representing all possible $\mathrm{CFC}^{\mathrm{PAC}}$ patterns across subjects and EEG segments). c) RM was reordered according to the three prototypes derived after applying VQ algorithm (see Fig.2a for the topographical layout). d) The time evolution of $\mu$ states $^{\mathrm{CFC}}$ has been demonstrated using the dinstinct colors for each $\mu$ states $^{\mathrm{CFC}}$ e so as timeintervals of constant microstate to be recognized. The height of colorbars correspond to the kurtosis estimated from the 64-tuples of PAC values.

\section{B. A codebook-network representation of transitions between ustates ${ }^{C F C}$}

The mined Net $\mu$ states for both tasks are all presented in Fig.3. Employing characteristic $\mu$ states $^{\mathrm{CFC}}$ for each condition, we attempted to describe the temporal evolution of the transition between $\mu$ states $^{\mathrm{CFC}}$. The proposed methodology transforms TVPAC (Fig.2.a) into a symbolic time series of $\mu$ states $^{\mathrm{CFC}}$ (Fig.2.d). By taking into account each symbolic time series for each task $\left(\mathrm{s}^{\mathrm{R}}(\mathrm{t})\right.$ and $\mathrm{s}^{\mathrm{AT}}(\mathrm{t}), \quad \mathrm{R}=$ Resting state and AT=Arithmetic Task), we estimated the observed probability Pobserved $(a, b)$ that in the symbolic time series $s^{\mathrm{R}, A T}(\mathrm{t}), \mathrm{i}=$ $1 \ldots, \mathrm{N}$ (where $\mathrm{N}$ denotes the \# of signal segments) symbol a is followed with symbol b. Transition dynamics between connectivity microstates is characterized by preferential transitions. Pobserved(a,b) is defined as:

$$
P_{\text {observed }}(a, b)=\frac{\# \text { of transitions from } a \text { to } b}{\text { \# of signal segments }-1}(3)
$$

The strength of the transition connections were estimated for each subject separately and finally group-averaged were presented. Figure 4 illustrates the codebook-network evolution with preferential transitions in both conditions weighted by $\mathrm{P}_{\text {observed. }}$.

Besides each $\mu$ states $^{\mathrm{CFC}}$ in Fig.3 the group-averaged fractional occupancy - FO is presented which is defined as the fraction of time spent in each state divide by the total $\mathrm{T}$ which denotes the length of the state sequence in samples $F O=\sum_{t=1}^{N} s_{i}^{R, A T}$

where $\mathrm{i}$ denotes one of the $\mu_{\text {states }}{ }^{\mathrm{CFC}}$.

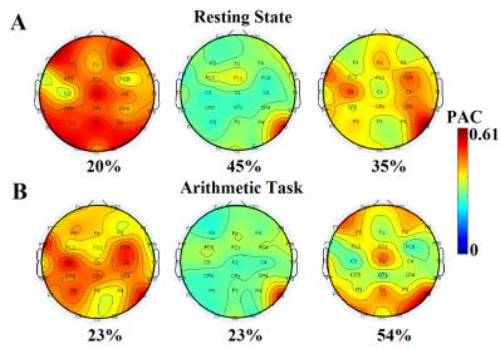

Figure 3. A topographic representation of $\mathrm{CFC}$ microstates $\left(\mu\right.$ states $^{\mathrm{CFC}}$ ) for both tasks. The percentage \% below each topography expresses the fraction of network organization patterns associated with it (in the ensemble formed from all subjects and signal segments).

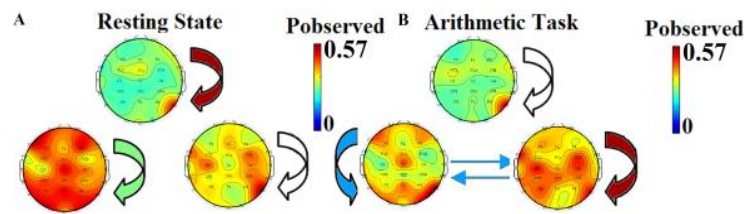

Figure 4. A network representation of the transition dynamics among CFC $\mu$ states for resting-state (a) and mental arithmetics (b). The color codes of arrow is related to $P_{\text {observed }}$ (to enhance visual inspection only the most important transitions are demonstrated with $\mathrm{P}_{\text {observed }}>0.1$ ). Self-loops reflect the constancy of CFC $\mu$ states.

\section{DISCUSSION}

We have presented a methodology for the efficient manipulation of nodal dynamic CFC in an adult group at two conditions: a resting-state and while performing a mental arithmetic task. The estimation of CFC was realized by a recently introduced measure called PAC [15,19]. The most important steps in the proposed methodology are the mining of nodal dynamic CFC via neural-gas algorithm [18], the induced symbolization (based on vector quantization) and the description of CFC dynamics through a restricted repertoire of states $^{\mathrm{CFC}}[7,21]$.

We adopted a recently introduced methodology which was first presented for the manipulation of time-varying functional connectivity graphs in a ERP study $[7,21]$. $\delta$ and $\gamma$ oscillations have a distinct spatial origin: $\delta$ oscillations are generated mainly anteriorly [23] whereas $\gamma$ oscillation reflect strictly local activity according to the task demands [24].Increased $\delta$ activity during cognitive tasks is an indicator of attentional task demands [25] while brain synchrony phenomena of $\gamma$ rhythm were related to processing of information that is independent of sensory modality [26]. It is evident that $\delta-\gamma$ CFC at resting state might play a key role in information integration [10]. Interestingly, by comparing with visual inspection $2^{\text {nd }}$ CFC $\mu$ states between tasks (Fig.3a vs Fig.3b), we can observed a complementary relation between them. Specifically, during resting wakefulness the default mode network (DMN) is active and is distributed over frontal and temporo-parietal brain regions (Fig.3 a) $-3^{\text {rd }}$ topography) $[25,27]$. During the mental task, brain areas related to the task are activated (mainly frontal, central and parieto-occipital 
brain regions) while the areas related to the resting-state were suppressed (Fig. $3 \mathrm{~b}$ ) $-3^{\text {rd }}$ topography). These findings can be explained as a deactivation of DMN and could reflect inhibitory mechanisms that are activated during cognitive mental arithmetic tasks [25,27]. During the addition task, several brain regions are active that supports functions such as extracting visual information (occipital cortex) or maintaining gaze fixation and the internal simulation of speech production (supplementary motor area and precentral cortex) [28,29]. Each hemisphere and especially the parietal lobe can recognize digits translating them into an abstract mental representation of quantities in order to add them $[28,29]$. The above findings revealed a possible organization role of $\delta$ activity that deactivate brain regions related to attentional demands and activate functions important for the execution of the task by a proper manipulation of $\gamma$ local activity. It would be interesting in the future to study these interactions in the source level and in relation with default-mode-network and fronto-paterial cognitive network at resting state and during cognitive tasks [30].

\section{Acknowledgment}

The authors would thank the National University of Singapore for supporting the Cognitive Engineering Group at the Singapore Institute for Neurotechnology (SINAPSE) under grant R-719-001-102-232, the DSO under the grant R-719-000-012-592 and MOE of Singapore under the grant MOE2014-T2-1-115.

\section{References}

[1] G.Deco, V. Jirsa, V. K. Robinson, P. A. Breakspear, and K.J.Friston, "The Dynamic brain: from spiking neurons to neural masses and cortical fields," PLoS Comput. Biol. 4:35, 2008.

[2] G.Deco, V. Jirsa, A.R. McIntosh, O. Sporns, and R.Kötter, "Key role of coupling, delay, and noise in resting brain fluctuations," Proc. Natl. Acad. Sci. U.S.A. vol.106, pp.10302-10307, April 2009.

[3] A.A.Ioannides, S.I.Dimitriadis, G.Saridis, M.Voultsidou, V.Poghosyan, L.Liu and N.A.Laskaris," Source Space Analysis of EventRelated Dynamic Reorganization of Brain Networks," Computational and Mathematical Methods in Medicine Volume 2012.

[4] C.J.Stam, B.F.Jones, G.Nolte, M.Breakspear and P.Scheltens," Small-world networks and functional connectivity in Alzheimer'sdisease," Cereb Cortex, vol.17, pp.92-99, 2007.

[5] S.Micheloyannis, M.Vourkas, V.Tsirka, E.Karakonstantaki, K.Kanatsouli and C.J.Stam," The influence of ageing on complex brain networks: A graph theoretical analysis. Hum Brain Mapp vol.30, pp.200-208, January 2012.

[6] S.I.Dimitriadis,N.A. Laskaris, V.Tsirka, M.Vourkas, S.Micheloyannis and S.Fotopoulos S," (2010b) Tracking brain dynamics via time-dependent network analysis," Journal of Neuroscience Methods vol.193,pp.145-155, October 2010b.

[7] S.I.Dimitriadis, N.A.Laskaris and A.Tzelepi," On the quantization of time-varying phase synchrony patterns into distinct Functional Connectivity Microstates (FC $\mu$ states) in a multi-trial visual ERP paradigm," Brain Topography vol.26,pp.397-409, July 2013a.

[8] S.I.Dimitriadis, N.A. Laskaris, P.G. Simos,S. Micheloyannis, J.J.Fletcher, R. Rezaie and A.C. Papanicolaou," Altered temporal correlations in resting-state connectivity fluctuations in children with reading difficulties detected via MEG," Neuroimage vol.83, pp.307-317, December 2013b.

[9] G.Buzsáki and B.O.Watson," Brain rhythms and neural syntax: implications for efficient coding of cognitive content and neuropsychiatric disease," Dialogues in Clinical Neuroscience vol.14,pp.345-67, December 2012

[10] V.Jirsa and V. Muller," Cross-frequency coupling in real and virtual brain networks," Front Comput Neurosci. vol.3,pp.7:78, July 2013
[11] Lehmann D, Faber PL, Galderisi S, Herrmann WM, Kinoshita T, Koukkou M, Mucci A, Pascual-Marqui RD, Saito N, Wackermann J, Winterer G \& Koenig T, "EEG microstate duration and syntax in acute, medicationnaïve, first-episode schizophrenia: a multi-center study" Psychiatry Res Neuroimaging vol.138, pp.141-156, 2005.

[12] F.De Vico Fallani, L.Astolfi, F.Cincotti, D.Mattia,M.G. Marciani, G.Gao, et al. Structure of the cortical networks during successful memory encoding in TV commercials," Clin Neurophysiol vol.119, pp.2231-2237, August 2008.

[13] S.I.Dimitriadis, N.A.Laskaris, A.Tzelepi and G.Economou," (2012b) Analyzing Functional BrainConnectivity by means of Commute Times: a new approach and its application to track event-related dynamics, "IEEE Transactions on Biomedical Engineering vol.59,pp.13021309, May 2012b.

[14] S.I.Dimitriadis,N.A. Laskaris,V.Tsirka, M.Vourkas, and S.Micheloyannis," An EEG study of brain connectivity dynamics at the resting state.,"Nonlinear Dynamics, Psychology and Life Sciences vol.16,pp.5-22, January 2012d.

[15] M.X.Cohen ," Assessing transient cross-frequency coupling in EEG data,"J. Neurosci. Methods vol.168, pp.494-499, March 2008.

[16] S. I. Dimitriadis, Y. Sun, K. Kwok, N. A. Laskaris, N. Thakor, and A. Bezerianos. Cognitive workload assessment based on the tensorial

treatment of eeg estimates of cross-frequency phase interactions. Ann.Biomed. Eng., 43(4):977-989, 2015.

[16] S. I. Dimitriadis, Y. Sun, K. Kwok, N. A. Laskaris, N. Thakor, and A. Bezerianos. Revealing cross-frequency causal interactions during a mental arithmetic task through symbolic transfer entropy: a novel vector-quantization approach. IEEE Trans Neural Syst Rehabil Eng. 2016 Jan 18. [Epub ahead of print]

[17] A.Delorme and S.Makeig," EEGLAB: An open source toolbox for analysis of single-trial EEG dynamics," J Neurosci Methods vol.134,pp.9-21, March 2004.

[18] Dimitriadis SI, Laskaris NA, Bitzidou MP, Tarnanas I and Tsolaki MN (2015) A novel biomarker of amnestic MCI based on dynamic crossfrequency coupling patterns during cognitive brain responses. Front. Neurosci. 9:350. doi: 10.3389/fnins.2015.00350

[19]J.Theiler, S.Eubank, A.Longtin, B.Galdrikian and D.Farmer," Testing for nonlinearity in time series: The method of surrogate data," Physica D vol.58, pp.77-94, September 1992.

[20]Dimitriadis SI, Laskaris NA, Micheloyannis S.Transition dynamics of EEG-based network microstates during mental arithmetic and resting wakefulness reflects task-related modulations and developmental changes. Cogn Neurodyn. 2015 Aug;9(4):371-87.

[21]T.Martinez, S.Berkovich and K.Schulten," Neural-gas network for vector quantization and its application to time-series prediction," IEEE Trans Neural Netw vol.4, pp.558-569, August 1993.

[22]Steriade, M., and Timofeev, I. (2003). Neuronal plasticity in thalamocortical networks during sleep and waking oscillations. Neuron 37, 563-576.

[23]Von Stein, A., Sarnthein, J., 2000. Different frequencies for different scales of cortical integration: from local gamma to long-range alpha/theta synchronization. Int. J.Psychophysiol. 38, 301-313.

[24]Dimitriadis SI, Laskaris NA, Tsirka V, Vourkas M, Micheloyannis S (2010a) What does delta band tell us about cognitive Processes: a mental calculation study. Neuroscience Letters 483:11-15.

[25]Tallon-Baudry, C., Bertrand, O., 1999. Oscillatory gamma activity in humans and its role in object representation. Trends Cogn. Sci. 3, 151-162.

[26]Buckner, R. L., Andrews-Hanna, J. R. and Schacter, D. L. (2008), The Brain's Default Network. Annals of the New York Academy of Sciences, 1124: $1-38$.

[27]S. Dehaene, M. Piazza, P. Pinel, L. Cohen, Three parietal circuits for number processing, Cogn. Neuropsychol. 20 (2003) 487-506.

[28]P. Pinel, S. Dehaene, D. Riviere, D. LeBihan, Modulation of parietal activation by semantic distance in a number comparison task,

NeuroImage 14 (2001) 1013-1026.

[29] S. I. Dimitriadis, N. A. Laskaris, S. Micheloyannis, "Transition dynamics of EEG-based network microstates during mental arithmetic and resting wakefulness reflects task-related modulations and developmental changes," Cogn. Neurodyn., vol. 9(4), pp. 371-87, Aug. 2015 